Pacific Journal of Mathematics CLOSED EXTENSIONS OF THE LAPLACE OPERATOR
DETERMINED BY A GENERAL CLASS OF BOUNDARY
CONDITIONS, FOR UNBOUNDED REGIONS 


\title{
CLOSED EXTENSIONS OF THE LAPLACE OPERATOR DETERMINED BY A GENERAL CLASS OF BOUNDARY CONDITIONS, FOR UNBOUNDED REGIONS
}

\author{
ROBERT S. FREEMAN
}

1. Introduction. In [1] certain closed operators in $L^{2}(G)$ were defined in terms of the Laplacian operator, and a class of generalized boundary conditions. There $G$ was a bounded domain in $E^{n}$ with $C^{1.1}$ boundary and the boundary conditions were of the form $\partial u / \partial n-L u=$ 0 , where $L$ was an arbitrary bounded operator on $L^{2}(\partial G)$. The operator so defined was denoted by $T_{L}$ and it was shown that $T_{L}$ was a closed linear operator with compact resolvent. Its adjoint $\left(T_{L}\right)^{*}$ was shown to be $T_{L *}$ and its spectrum was shown to lie inside a parabola of the form

$$
y^{2}=a x+b \text { where } a, b>0 .
$$

It is the purpose of this paper to extend the results of [1] by removing the restriction that $G$ be bounded. The previous history of the problem is adequately covered in [1]. It is worth noting here that the underlying idea of [1] derives from a theorem of Calkin [4] and relies on the compactness of the resolvent of an operator $S$ on $L^{2}(G) \oplus L^{2}(\partial G)$. For certain of the results of [1] it is only necessary to assume the closedness of the range of $S$ but many of the results explicitly used the compactness of the resolvent. If $G$ is unbounded, the resolvent of $S$ is no longer compact and the methods of [1] can no longer be used.

The approach taken here is to use localization methods to reduce the problem for unbounded regions to one for bounded regions. We observe that the Laplacian is an operator of local type in the sense of Hörmander [7]. However in applying these methods we must distinguish between two cases. If $\partial G$ is bounded we can separate the behavior of functions near the boundary from their behavior near infinity by multiplying them by functions which vanish in a neighborhood of infinity and are identically one near the boundary. When $\partial G$ is unbounded this can no longer be done and any such attempt at localization must affect the behavior of the function on portions of the boundary. It turns out to be necessary to require that any localization function, regarded as an operator on $L^{2}(\partial G)$, must commute with the boundary operator $L$. For this reason when $\partial G$ is unbounded we restrict our attention to

Received February 20, 1961. This paper is a development of portions of the author's doctoral thesis done under the supervision of Professor W. G. Bade and submitted to the University of California, Berkeley, California. The author wishes at this time to express his gratitude to Professor Bade for his advice and encouragement and for reading and criticizing this manuscript. 
multiplication operators on the boundary.

It is our hope for the future to extend these results to uniformly elliptic, second-order operators on $L^{p}(G)$ where $1<p<\infty$.

In $\S 1$ we list some gєometric preliminaries. In $\S 2$ we list results centering about the Lax-Milgram theorem [8] and preliminary localization results. The main results are contained in $\S \S 3$ and 4 . In $\S 3$ we treat operators for unbounded regions with bounded boundaries and in $\S 4$ we treat the situation where the boundary is unbounded.

1. Preliminaries. In what follows $G$ will be a possibly unbounded open connected set in $E^{n}$. Its boundary will be denoted by $\partial G$. Points in $G$ will be denoted by $x, y, \cdots$ and points in $\partial G$ will be denoted by $\theta, \phi, \cdots$. We will denote by $d x, d y, \cdots$ and by $d \theta, d \phi, \cdots n$-dimensional and $n-1$ dimensional Lebesgue measures on $G$ and $\partial G$, respectively. The exterior unit normal to $\partial G$ at $\theta$ will be denoted by $n_{\theta}$. As in [1] we will always assume that $\partial G$ is an $n-1$ dimensional $C^{1,1}$ manifold. A detailed treatment of such manifolds may be found in Lucas [9]. For convenience we will state the fundamental

1.1. THEOREM. Let $\Gamma$ be a $C^{1,1}$ manifold, then there exists a positive number, $r_{0}$, called the minimal radius of $\Gamma$ such that:

(i) All segments of length $r_{0}$ centered at points of $\Gamma$ and normal to $\Gamma$ are mutually disjoint and exhaust a neighborhood of $I^{\prime}$, called an $r_{0}$ neighborhood of $\Gamma$.

(ii) At each point $\theta$ in $\Gamma$, spheres interior and exterior to $\Gamma$ of radius $r_{0}$ may be drawn tangent to $\Gamma^{\top}$ at $\theta$ and containing no other points of $\Gamma$.

(iii) The normals to $\Gamma$ satisfy a uniform Lipschitz condition with Lipschitz constant $r_{0}^{-1}$, i.e.,

$$
\left|n_{\theta}-n_{\phi}\right| \leqq r_{0}^{-1}|\theta-\phi|
$$

for $\phi, \theta \in \partial G$.

(iv) The set $\Gamma_{\rho}=\left\{\theta-\rho n_{\theta}: \theta \in \Gamma\right\}$ is a $C^{1,1}$ manifold for $\rho<r_{0}$ with minimal radius $r_{\mathrm{\rho}}$ satisfying $r_{0}-|\rho| \leqq r_{\mathrm{\rho}} \leqq r_{0}+|\rho|$. Such surfaces will be called parallel surfaces to $\Gamma$ and are denoted by $\Gamma_{\rho}$.

$(\mathrm{v})$ The surface measures on $\Gamma$ and $\Gamma_{\rho}$ are mutually absolutely continuous with uniformly bounded Radon-Nikodym derivatives for $|\rho|<r_{0} / 2$.

We impose further restrictions on $\partial G$. We require that there exists a covering of every $r$ neighborhood of $\partial G$, for $r<r_{0}$, by a possibly finite sequence of open sets $\left\{V_{k}\right\}$ such that

(a) For each $k$ there exists a homeomorphism, $T_{k}$ of $V_{k}$ into $E^{n}$ such that $T_{k}\left(V_{k} \cap G\right)=\Gamma \times\left\{0<x_{n}<r\right\}$ where $\Gamma$ is an $n-1$ dimensional 
sphere with center at the origin and diameter $r$.

(b) $T_{k}, T_{k}^{--1}$ and their derivatives up to order $j$ satisfy a uniform Lipschitz condition.

(c) There exists an integer $N$ such that at most $N$ of the sets $\left\{V_{k}\right\}$ have a nonempty intersection.

(d) $\bigcup_{k} T_{k}^{-1}\left\{1 / 2 \Gamma x\left[0<x_{n}<r / 2\right]\right\}$ covers an $r / 2$ neighborhood of $\partial G$.

1.2. Definition. If $\partial G$ satisfies conditions $(a)-(d)$ above we say that $G$ is a uniformly $C^{j, 1}$ domain.

Usually $j$ can be 1 , however later in $\S 4$ we shall need $j$ to be two. If the derivatives up to order $j$ of $T_{k}, T_{k}^{-1}$ are merely continuous and bounded, we speak of a uniformly $C^{j}$ domain.

In addition to the function space $L^{2}(G)$ we refer to certain other function spaces which are by now quite familiar. The space $C_{0}^{\infty}(G)$ is the set of all infinitely differentiable functions with compact support in $G$. We shall always consider complex valued functions unless stated to the contrary. In the notation of Browder [2], $W^{j, p}(G)$ is the set of all functions in $L^{p}(G)$ whose distribution valued derivatives up to order $j$ are functions in $L^{p}(G)$. Using the familiar notation

$$
\alpha=\left\langle\alpha_{1}, \cdots, \alpha_{n}\right\rangle \text { and }|\alpha|=\sum_{i=1}^{n} \alpha_{i}
$$

where the $\alpha_{i}$ are nonnegative integers, we write

$$
D^{\alpha}=\frac{\partial^{\alpha_{1}}}{\partial x_{1}^{\alpha_{1}}} \cdots \frac{\partial^{\alpha_{n}}}{\partial x_{n}^{\alpha_{n}}}
$$

for the generic differential operator of order $|\alpha|$. For $u \in W^{j, p}(G)$ we write

$$
\|u\|_{j, p}=\left(\sum_{|\alpha| \leqq j}\left\|D^{\alpha} u\right\|_{p}^{p}\right)^{1 / p}, \quad 1<p<\infty
$$

where $\|\cdot\|_{p}$ is the $L^{p}$ norm. Here we shall mainly be concerned with the $L^{2}$ norm which we shall write as $\|\cdot\|$. The following well known facts we state as a

1.3. Lemma. $W^{j, p}(G)$ is a Banach space in the norm $\|\cdot\|_{j, p}$, and a Hilbert space for $p=2 . C_{0}^{\infty}\left(E^{n}\right)$ is dense in $W^{j, p}(G)$ in the $\|\cdot\|_{j, p}$ norm.

Let $\Gamma$ be a subset of $\partial G$. For $0<\rho<r_{0}$ and for $\phi \in \Gamma$ define the set $G_{\rho}$ to be $\left\{x \in G: x=\phi-\rho n_{\phi}\right\}$. For fixed $\rho<r_{0}$ let $\Gamma_{\rho}=\{x \in G: x=$ $\left.\phi-\rho n_{\phi}, \phi \in \Gamma\right\}$. If $u$ is a function in $W^{1, p}\left(G_{\rho}\right)$ we write $u_{\rho}(\phi)=u(\phi-$ $\left.\rho m_{\phi}\right), \phi \in \Gamma$. Thus $u_{\rho}$ is the restriction of $u$ to $\Gamma_{\rho}$. We now make the following definition 
1.4. Definition. $u_{\rho}$ has an $L^{p}$-boundary value on $I$ if

(i) $u_{\rho} \in L^{p}\left(\Gamma_{\rho}\right)$ for $\rho<r_{0}$.

(ii) There exists a function $\widetilde{u} \in L^{p}(\Gamma)$ such that

$$
\lim _{\rho \rightarrow 0} \int_{\Gamma}\left|u_{\rho}(\phi)-\tilde{u}(\phi)\right|^{p} d \phi=0 .
$$

We now introduce the class of functions with which we shall be dealing throughout the remainder of this paper.

1.5. Definition. $\mathscr{D}_{1}(G)$ is the set of all functions $u$ in $W^{1,2}(G)$ such that

(i) $u \in W^{2,2}\left(G_{1}\right)$ for each open set $G_{1}$ whose closure is compact and lies in $G$,

(ii) $\Delta u \in L^{2}(G)$,

(iii) $u$ has an $L^{2}$ boundary value $\tilde{u}$ on $\partial G$, and $\partial u / \partial n$ has an $L^{2}$ boundary value on every compact subset $\Gamma$ of $\partial G$.

It is well known [5] that every function in $W^{1,2}$ has an $L^{2}$ boundary value and an examination of harmonic functions on the unit circle shows that the inclusion $W^{2,2}(G) \subseteq D_{1}(G) \subseteq W^{1.2}(G)$ is proper.

Recall that the "minimal operator" associated with the Laplacian is the closure of the Laplacian restricted to $C_{0}^{\infty}(G)$ and the "maximal operator" is its adjoint. Its domain is the set of functions in $W^{12}(G)$ satisfying conditions (i) and (ii) of Definition 1.5.

2. Lax-Milgram theorem. We here present the Lax-Milgram theorem in a form suitable for our purposes. It is the generalization of the Friedrichs' extension theorem [6] to the nonsymmetric case.

Let $\mathscr{K}$ and $\mathscr{H}_{1}$ be two Hilbert spaces with norms $\|\cdot\|$ and $\|\cdot\|_{1}$, respectively. We suppose

(i) $\mathscr{K}_{1}$ is densely contained in $\mathscr{H}$.

(ii) There exists a number $k>0$ such that for all $u$ in $\mathscr{K}_{1}$

$$
k\|u\| \leqq\|u\|_{1} .
$$

Let $B$ be a not necessarily Hermitian symmetric bilinear form whose domain is all of $\mathscr{H}_{1}$ and suppose

$$
|B(u, v)| \leqq K\|u\|_{1}\|v\|_{1} \text { for } u, v \in \mathscr{H}_{1}
$$

$\operatorname{Re} B(u, u) \geqq C\|u\|_{1}^{2}$ for $u \in \mathscr{H}_{1}$.

Lax and Milgram dealt with real Hilbert spaces whereas here and throughout this paper we deal with complex Hilbert spaces. Condition (b) is the obvious modification of their condition, and the necessary changes to their proof resulting from (b) are of a trivial nature. 
2.1. THEOREM (Lax-Milgram). For any bounded linear functional $l$ on $\mathscr{H}_{1}$ there exist two vectors $v, v^{*}$ in $\mathscr{H}_{1}$ such that

(i) $l(u)=B(u, v)=B\left(v^{*}, u\right)$ for $u \in \mathscr{H}_{1}$.

(ii) If $w \in \mathscr{H}_{1}$ and $l=(\cdot, w)_{1}$ and $v=P_{1} w$, then $P_{1}$ is a linear homeomorphism of $\mathscr{H}_{1}$ onto $\mathscr{H}_{1}$.

(iii) If $U$ is a proper closed subspace of $\mathscr{K}_{1}$, then there exists a $v \in \mathscr{Y}_{1}$ such that for every $u \in U, B(u, v)=0$.

2.2. THEOREM. There exists a closed densely defined linear transformation $T$ of $\mathscr{H}$ into itself such that $T$ and its adjoint $T^{*}$ have domains contained in $\mathscr{H}_{1}$ and have bounded everywhere defined inverses in $Y$. Moreover

$$
B(u, v)=(u, T v) \text { for } u \in \mathscr{H}_{1}, v \in O(T)
$$

and

$$
B(u, v) \quad\left(T^{*} u, v\right) \text { for } u \in \mathscr{M}\left(T^{*}\right), v \in \mathscr{H}_{1} .
$$

The fact the $T$ is densely defined in $\mathscr{C}^{\prime}$ and closed as a map of $\mathscr{H}$ into $\mathscr{C}$ was not explicitly stated in Lax-Milgram. The density follows from the fact that $T^{-1}$ is everywhere defined in $\mathscr{C}$ and bounded. In fact if $z$ is orthogonal to $\mathscr{X}(T)$ then for $u \in \mathscr{D}(T),(u, z)=0$ and since $(., z)$ is a bounded linear functional on $\mathscr{H}_{1}$ and $\mathscr{D}(T) \subseteq \mathscr{H}_{1}$ it follows that $(u, z)=B\left(u, T^{-1} z\right)=0$ for $u \in \mathscr{D}(T)=\mathscr{R}\left(T^{-1}\right)$. Thus $B\left(T^{-1} u, T^{-1} z\right)=$ 0 and in particular $B\left(T^{-1} z\right)^{1}=0$ and thus $T^{-1} z=0$. Hence $z=0$. To show that $T$ is closed as a map of $\mathscr{E}$ into $\mathscr{K}$, let $\left\{v_{n}\right\}$ be a sequence in $\mathscr{D}(T)$ and suppose $v_{n} \rightarrow v \in \mathscr{C}$ and $T v_{n} \rightarrow w \in \mathscr{C}$. Now for each $n$ and all $u \in \mathscr{C}_{1} B\left(u, v_{n}\right)=\left(u, T v_{n}\right)$ and thus $B\left(v_{n}\right)=\left(v_{n}, T v_{n}\right) \rightarrow(v, w)$. But then $v_{n}$ converges in $\mathscr{H}_{1}$ to say $z$ and thus in $\mathscr{H}$. Hence $v=z$. Since $T$ is closed as a map of $\mathscr{K}_{1}$ into $\mathscr{H}, T z=w=T v$.

The facts about $T^{*}$ follow easily if one recalls that $T^{-1}$ is everywhere defined and bounded.

We shall now apply Theorem 2.2 to a study of the Laplacian operator, considered as an operator on $L^{2}(G)$. With the aid of Theorem 2.2 we shall prove the existence of a closed densely defined operator with domain in $W^{1,2}(G)$ and satisfying certain boundary conditions in a generalized sense. Since the domains lie in $W^{1,2}(G)$ we know that the functions have $L^{2}$ boundary values. It will then be the task of the remainder of this paper to show in what sense the boundary conditions are satisfied. To begin we require the following

2.3. Lemma. Let $G$ be a uniformly $C^{1.1}$ manifold. Then for any $u \in W^{1,2}(G), u$ has an $L^{2}$ boundary value $\tilde{u}$ and for each $\varepsilon>0$, there exists a $C(\varepsilon)>0$ such that $\|\tilde{u}\|_{a}^{3} \leqq K\left[\varepsilon\|\nabla u\|^{2}+C(\varepsilon)\|u\|^{2}\right]$.

1 We write $B(x)$ for $B\left(x_{j}, x\right)$. 
Here $\nabla$ represents the gradient of $u$ and $\|\cdot\|_{\partial}$ is the norm on $L^{2}(\partial G)$.

This lemma appears widely in the literature, see for example Ehrling [5] and Nirenberg [10] where $G$ is assumed to be bounded. When $G$ is unbounded the proof can be suitably modified without too much difficulty with the aid of a partition of unity. For an outline of this proof see Poulsen [11].

Suppose $u$ is a smooth function and that $L$ is a bounded linear operator on $L^{2}(\partial G)$ and that $\widetilde{u}_{n}-L \widetilde{u}=0$. Let

$$
\int_{\theta} \nabla u \cdot \overline{\nabla v} d x=\int_{\theta} \sum_{i=1}^{n} \frac{\partial u}{\partial x_{i}} \frac{\overline{\partial v}}{\partial x_{i}} d x
$$

and apply Green's formula to obtain

$$
(-\Delta u, u)=\|\nabla u\|^{2}-(L \widetilde{u}, \widetilde{u})_{\partial} .
$$

2.4. Definition. For $u \in W^{1,2}(G)$ let

$$
\begin{gathered}
B_{G}(u)=\|\nabla u\|^{2}-(L \tilde{u}, \tilde{u})_{\partial} \\
B_{G}^{\gamma}(u)=B_{G}(u)+\gamma\|u\|^{2}
\end{gathered}
$$

where $\gamma$ is any complex number. Let $L$ be any bounded operator on a Hilbert space $\mathscr{H}$. Form $L_{1}=1 / 2\left(L+L^{*}\right)$ and $L_{2}=1 / 2\left(L-L^{*}\right)$ so that $L_{1}^{*}=L_{1}, L_{2}^{*}=-L_{2}$ and $L=L_{1}+L_{2}$. Moreover, $R_{l}(L u, u)=\left(L_{1} u, u\right)$ and $\operatorname{Im}(L u, u)=1 / i\left(L_{2} u, u\right) . \quad L_{1}$ and $L_{2}$ are respectively the symmetric and antisymmetric parts of $L$.

2.5. LEMma. If the real part of $\gamma$ is sufficiently large, then for all $u \in W^{1,2}(G)$

$$
R_{l} B_{G}^{\gamma}(u) \geqq \frac{1}{2}\|u\|_{1,2}^{2} .
$$

Proof. Let $R_{l} \gamma=\alpha$. Then

$$
\begin{aligned}
R_{l} B_{G}^{\gamma}(u) & =\|\nabla u\|^{2}+\alpha\|u\|^{2}-\left(L_{1} \tilde{u}, \tilde{u}\right)_{\partial} \\
& \geqq\|\nabla u\|^{2}+\alpha\|u\|^{2}-\left\|L_{1}\right\|\|\tilde{u}\|_{\partial}^{2} .
\end{aligned}
$$

Now by Lemma 2.3

$$
\begin{aligned}
R_{l} B_{G}^{\gamma}(u) & \geqq\|\nabla u\|^{2}+\alpha\|u\|^{2}-\left\|L_{1}\right\| K\left[\varepsilon\|\nabla u\|^{2}+C(\varepsilon)\|u\|^{2}\right] \\
& =\left(1-\left\|L_{1}\right\| K \varepsilon\right)\|\nabla u\|^{2}+\left(\alpha-\left\|L_{1}\right\| K C(\varepsilon)\right)\|u\|^{2} .
\end{aligned}
$$

Choose $\varepsilon$ so small that $1-\left\|L_{1}\right\| K \varepsilon>1 / 2$ and $\alpha$ so large that

$$
\alpha-\left\|L_{1}\right\| K C(\varepsilon)>\frac{1}{2} \text {. }
$$

Note that when $L$ is the zero operator $\gamma$ may be taken to be real, 
for the form $B_{G}$ is symmatric.

2.6. Lemma. There exists a positive real number $C$ such that for every $u$ and $v \in W^{1,2}(G)$

$$
\left|B_{f}^{\gamma}(u, v)\right| \leqq C\|u\|_{1}\|v\|_{1}
$$

Proof.

$$
\begin{aligned}
\left|B_{G}^{\gamma}(u, v)\right| \leqq & \left|\int_{G} \nabla u \cdot \overline{\nabla v} d x\right|+|\gamma(u, v)|+\left|(L u, v)_{\partial}\right| \\
\leqq & \|\nabla u\|\|\nabla v\|+|\gamma|\|u\|\|v\|+\|L\|\|u\|_{\partial}\|v\|_{\partial} \\
\leqq & \|\nabla u\|\|\nabla v\|+|\gamma|\|u\|\|v\|+\|L\|\left[K \left(\varepsilon\|\nabla u\|^{2}\right.\right. \\
& \left.\left.\left.+C(\varepsilon)\|u\|^{2}\right)\right]^{1 / 2} \mid K\left(\varepsilon\|\nabla u\|^{2}+C(\varepsilon)\|v\|^{2}\right)\right]^{1 / 2}
\end{aligned}
$$

for any $\varepsilon>0$. Choose $\varepsilon>0$ and let $\delta=\max (\varepsilon, C(\varepsilon))$. Let $\eta=$ $\max (|\gamma|, 1)$ and $C=2 \eta+\delta\|L\|$. Then a simple computation yields (2.6.1).

Let $\mathscr{Y}=L^{2}(G)$ and $\mathscr{Y}_{1}=W^{1,2}(G)$ and apply the Lax-Milgram theorem to the form $B_{q}^{\gamma}$. The result is the following:

2.7. Theorem. Let $L$ be any bounded linear operator on $L^{2}(\partial G)$. Then there exists a closed, densely defined, linear operator $M_{L}$ in $L^{2}(G)$ with domain in $W^{1,2}(G)$ sush that for every $v \in \mathscr{D}\left(M_{L}\right)$ and $u \in W^{1,2}(G)$, $B_{G}(u, v)=\left(u, M_{L} v\right)$. Its adjoint $M_{L}^{*}$ enjoys the relation $B_{G}(u, v)=$ $\left(M_{L}^{*} u, v\right)$ for $u \in \mathscr{D}\left(M_{L}^{*}\right)$ and $v \in W^{1,2}(G)$. Moreover, if $\gamma$ is any complex number such that $R_{l} \gamma \leqq-\left(\delta+\left\|L_{1}\right\| K C(\varepsilon)\right)$ where $0<\delta<1$ and $C(\varepsilon)$ is the number of Lemma 2.3 with $\varepsilon<(1-\delta) /\left\|L_{1}\right\|$, then $\gamma$ lies in the resolvent set of $M_{L}$.

Proof. When $\gamma$ is as above, Theorem 2.2 shows that $M_{L}+\gamma I$ is closed and densely defined in $L^{2}(G)$ with domain in $W^{1,2}(G)$. Moreover, zero is in its resolvent sat.

We shall now give a characterization of $\mathscr{D}\left(M_{L}\right)$.

2.8. THEOREM. Let $v \in W^{1,2}(G)$, then a necessary and sufficient condition that $v \in \mathscr{D}\left(M_{L}\right)\left(\mathscr{D}\left(M_{L}^{*}\right)\right)$ is that $-\Delta v$ is a distribution in $L^{2}(G)$, and for $u \in W^{12}(G),(u,-\Delta v)=B(u, v)((-\Delta v, u)=B(v, u))$.

Proof. We give the proof for $M_{L}$, the proof for $M_{L}^{*}$ being virtually identical.

Lat $v \in \mathscr{D}\left(M_{L}\right)$. Then for every $u \in W^{1,2}(G)$, and hence for every $u$ in $C_{v}^{\infty}(G),\left(u, M_{L} v\right)=B(u, v)$. Recalling the definition of the derivative of a distribution, we have $\left(u, M_{L} v\right)=B(u, v)=\int_{G} \nabla u \cdot \overline{\nabla v} d x=(u,-\nabla v)$ 
for $u$ in $C_{0}^{\infty}(G)$. Since $C_{0}^{\infty}(G)$ is dense in $L^{2}(G)$ it follows that $M_{L} v=$ $-\Delta v$. Now suppose $v$ is in $W^{1,2}(G)$ and $-\Delta v$ is a distribution in $L^{2}(G)$ such that for $u \in W^{1,2}(G),(u, \Delta v)=B_{G}(u, v)$. Then for a suitable complex number $\gamma$ the form $B_{G}^{\gamma}$ defined by $(u,-\Delta v+\gamma v)=B_{G}^{\gamma}(u, v)$ is bounded in $W^{1,2}(G)$ and $R_{l} B_{G}^{\gamma}$ is bounded from below. It then follows from the proof of Theorem 2.2 that $v \in \mathscr{D}\left(M_{L}\right)$ and $M_{L} v=-\Delta v$.

We now present a series of localization lemmas which will be used in what follows. These results are by now quite common in the literature and we follow for the most part the presentation given in Poulsen [11].

2.9. Lemma. Let $\omega$ be in $C^{1}(G)$ and suppose $K$ is an upper bound for $\omega$ and $|\nabla \omega|$. Then for every $u \in W^{1,2}(G)$, $\omega u$ is also in $W^{1,2}(G)$.

Proof. $W^{1,2}(G)$ is the closure of $C_{0}^{1}\left(E^{n}\right)$ in the $W^{1,2}$ norm. If $\left\{u^{j}\right\}$ is a sequence in $C_{0}^{1}\left(E^{n}\right)$ such that $\left\|u^{j}-u\right\|_{1,2} \rightarrow 0$. Then the inequalities $\left\|\omega u^{j}-\omega u\right\| \leqq K\left\|u^{j}-u\right\|$ and $\left\|\nabla\left(\omega u^{j}\right)-\nabla(\omega u)\right\| \leqq K\left(\left\|u^{j}-u\right\|+\right.$ $\left.\left\|\nabla u^{j}-\nabla u\right\|\right)$ show that $\left\|\omega u^{j}-\omega u\right\|_{1,2} \rightarrow 0$.

2.10. Lemma. Let $\omega \in C_{0}^{1,1}\left(E^{n}\right)$ be real valued and suppose that $K$ is an upper bound for $|\omega|,|\nabla \omega|$, and $|-\Delta \omega|$ in $G$. If $u \in W^{1,2}(G)$ and $-\Delta u$ is a distribution in $L^{2}(G)$ then $\omega u \in W^{1,2}(G)$ and $-\Delta(\omega u)$ is a distribution in $L^{2}(G)$.

Proof. Let $S(u, \omega)=(-\Delta \omega) u-2 \nabla \omega \cdot \nabla u-\omega \Delta u$ and observe that

$$
\|S(u, \omega)\| \leqq K(\|u\|+2\|\nabla u\|+\|\Delta u\|) .
$$

Thus $S(u, \omega)$ is clearly a distribution in $L^{2}(G)$ and by the definition of derivative of a distribution $S(\omega, u)=-\Delta(\omega u)$.

3. Regions with bounded boundaries. In the preceding section we have given a characterization of the operator $M_{L}$ in terms of the Laplacian and the form $B_{G}(\cdot, \cdot)$. Hereafter the symbol $M_{L}$ will always stand for this operator. Essentially these results concern themselves with regularity in the interior of $G$. It is the purpose of this section to study the boundary behavior of functions in $\mathscr{D}\left(M_{L}\right)$. Explicitly let $\mathscr{D}\left(T_{L}\right)$ be the set of $u \in \mathscr{D}_{1}(G)$ (cf. Definition 1.5) for which $\widetilde{u}_{n}-L \widetilde{u}=0$ and let $T_{L}$ be $-\Delta$ with domain $\mathscr{D}\left(T_{L}\right)$, then we shall show $T_{L}=M_{L}$.

3.1. Definition. Let $\phi$ be a function in $C_{0}^{\infty}\left(E^{n}\right)$ satisfying

$$
\phi(x)= \begin{cases}1 & |x| \leqq 1 \\ 0 & |x| \geqq 2\end{cases}
$$


and $0 \leqq \phi(x) \leqq 1$. Let $\omega^{N}(x)=\phi(x / N)$ and let $K$ be an upper bound for $|\nabla \phi|$ and $|\Delta \phi|$.

\subsection{LEMMA.}

$$
\begin{aligned}
& \left|\nabla \omega^{N}\right| \leqq K / N \\
& \left|\Delta \omega^{N}\right| \leqq K / N^{2} .
\end{aligned}
$$

3.3. LEMmA. Let $u \in W^{1,2}(G)$ and suppose - $u$ is a distribution in $L^{2}(G)$. Then as $N \rightarrow \infty$

$$
\begin{gathered}
\left\|\omega^{N} u-u\right\|_{1,2} \rightarrow 0 \\
\left\|-\Delta\left(\omega^{N} u\right)-(-\Delta u)\right\| \rightarrow 0 .
\end{gathered}
$$

Proof. For each $N, \omega^{N} u \in W^{1,2}$ and $-\Delta\left(\omega^{N} u\right)$ is a distribution in $L^{2}(G)$ by Lemma 2.10. The results follow from an obvious computation, Lemma 3.2, and the dominated convergence theorem.

3.4. THEOREM (Green's Formulas). Let $u$ and $v$ be in $\mathscr{D}_{1}(G)$. Then

$$
\begin{gathered}
\int_{G}(-\Delta u) \bar{v} d x=\int_{G} \nabla u \cdot \overline{\nabla v}-\int_{G \partial} \widetilde{u}_{n} \overline{\widetilde{v}} d \theta \\
\int_{G}(-\Delta u) \bar{v} d x-\int_{G} u(-\overline{\Delta v}) d x=\int_{\partial G}\left(\tilde{u}_{\tilde{v}_{n}}-\widetilde{u}_{n} \overline{\widetilde{v}}\right) d \theta .
\end{gathered}
$$

Proof. Let $B_{N}$ be the sphere with center at the origin and radius $N$ and let $\omega^{N}$ be the function of Lemma 3.2. Define $v^{N}$ to be $\omega^{N} v$ so that $v$ vanishes outside of $B_{2 N}$. Then

$$
\begin{aligned}
\int_{G}(-\Delta u) \bar{v}^{N} d x= & \int_{B_{3 N} \cap G}(-\Delta u) \overline{\widetilde{v}^{N}} d x=\int_{B_{3 N} \cap G} \nabla u \cdot \overline{\nabla v^{N}} d x \\
& -\int_{\partial B_{3} N \cup \partial G} \widetilde{u}_{n} \overline{\widetilde{v}^{N}} d \theta \\
= & \int_{G} \nabla u \cdot \overline{\nabla v^{N}} d x-\int_{\partial G} \tilde{u}_{n}{\overline{\tilde{v}^{N}}} d \theta .
\end{aligned}
$$

The last of the equalities follows from the fact that $\omega^{N}$ vanishes on and near $\partial B_{3 N} \cdot{ }^{2}$ Let $N \rightarrow \infty$ and apply Lemma 3.3 to obtain Eq. (3.4.1). Formula (3.4.2) can be obtained in a similar manner.

3.5. THEOREM (Localization). Let $\omega^{N}$ be as in 3.1. If $v \in \mathscr{D}\left(M_{L}\right)\left(\mathscr{D}\left(M_{L}^{*}\right)\right)$, then $\omega^{N} v \in \mathscr{D}\left(M_{L}\right)\left(\mathscr{D}\left(M_{L}^{*}\right)\right)$.

Proof. We give the proof for $M_{L}$. By Theorem 2.8 and Lemma 2.10 it suffices to show that for $u \in W^{1,2}(G)$ or even for $u \in C_{0}^{2}(G)$

${ }^{2}$ Observe that $\tilde{v}^{N}=\tilde{v}$ on $\partial G$. 


$$
\left(u,-\Delta\left(\omega^{N} v\right)\right)=B\left(u, \omega^{n} v\right)
$$

Here of course $N$ is assumed to be sufficiently large that $\partial G \cong B_{N}$. We note that $C_{0}^{2}\left(E^{n}\right)$ is dense in $W^{1,2}(G)$ and, moreover, that if $\left\{u^{j}\right\}$ is a sequence in $C_{0}^{2}\left(E^{n}\right)$ such that $\left\|u^{j}-u\right\|_{1,2} \rightarrow 0$ and if $\widetilde{u}^{j}$ is the corresponding boundary value, then $\left\|\widetilde{u}^{j}-\tilde{u}\right\|_{\partial} \rightarrow 0$. Hence it suffices to prove (3.5.1) for $u \in C_{0}^{2}\left(E^{n}\right)$. Now by Lemma 2.10, for all $u \in W^{1,2}(G)$

$$
(u,-\Delta(\omega v))=(u, \omega(-\Delta v))+(u, v(-\Delta \omega))-2 \int_{G} u \nabla \overline{\omega \cdot \nabla v} d x
$$

since $\omega$ is real valued. Now

$$
(u, \omega(-\Delta v))=\int_{G} u \omega(-\overline{\Delta v}) d x=B_{G}(u \omega, v) .
$$

Moreover,

$$
(u, v(-\Delta \omega))=\int_{G} u \bar{v}(-\Delta \omega) d x=\int_{\Omega} u \bar{v}(-\Delta \omega) d x=\int_{\Omega} \nabla(u \bar{v}) \cdot \nabla \omega d x .
$$

Here $\Omega=G \cap B_{3 N}$ and we note that $\omega$ vanishes on and near $\partial B_{3 N}$. Thus

$$
(u,-\Delta(\omega v))=B_{G}(u \omega, v)+\int_{G} \nabla(u \bar{v}) \cdot \nabla \omega d x-2 \int_{G} u \nabla \omega \cdot \overline{\nabla v} d x .
$$

Since $B_{G}(u \omega, v)=\int_{\theta} \nabla(u \omega) \cdot \overline{\nabla v} d x-\int_{\partial G} u \omega \overline{L v} d x$ and $\omega=1$ on $\partial G$ and

$$
\begin{aligned}
\int_{G} \nabla(u \omega) \cdot \overline{\nabla v} d x & +\int_{G} \nabla(u \bar{v}) \cdot \nabla \omega d x-2 \int_{G} u \nabla \omega \cdot \overline{\nabla v} d x \\
& =\int_{G} \omega \nabla u \cdot \overline{\nabla v} d x+\int_{G} \nabla u \cdot \nabla \omega \bar{v} d x=\int_{G} \nabla u \cdot \nabla(\omega v) d x,
\end{aligned}
$$

we have finally

$$
(u,-\Delta(\omega v))=B(u, \omega v) \text { for } u \in C_{0}^{2}\left(E^{n}\right) .
$$

3.6. Definition. Let $\mathscr{D}\left(T_{L}\right)\left(\mathscr{D}\left(T_{L *}\right)\right)$ be the set of those $u \in \mathscr{D}_{1}(G)$ for which $\widetilde{u}_{n}-L \widetilde{u}=0\left(\widetilde{u}_{n}-L^{*} \widetilde{u}=0\right)$ and for $u$ in $\mathscr{D}\left(T_{L}\right)\left(\mathscr{D}\left(T_{L *}\right)\right)$, let $T_{L} u\left(T_{L *} u\right)=-\Delta u$.

When $G$ was bounded it was shown in [1] that $T_{L}$ was closed and densely defined and that $\left(T_{L}\right)^{*}=T_{L *}$. In the remainder of this section we shall identify $M_{L}$ with $T_{L}$ and $M_{L}^{*}$ with $T_{L *}$, thus proving that for $G$ unbounded, but with $\partial G$ bounded, we again have $\left(T_{L}\right)^{*}=T_{L *}$.

It is immediately clear by Green's formula that $T_{L} \subseteq M_{L}$ and $T_{L *} \subseteq$ $M_{L}^{*}$. The reverse inclusions will be obtained by localization. Consider a sphere $B_{3 N}$ with $N$ so large that $\partial G \leqq B_{N}$, and let $\Omega=G \cap B_{3 N}$, so that $\partial \Omega=\partial G \cup \partial B_{3 N}$. We define an operator, $L_{0}$ on $L^{2}(\partial \Omega)$ by 


$$
L_{0} \widetilde{u}= \begin{cases}L \tilde{u} & \tilde{u} \in L^{2}(\partial G) \\ 0 & \tilde{u} \in L^{2}\left(\partial B_{3 N}\right)\end{cases}
$$

and the operator $T_{L_{0}}$ analogously as in Definition 3.6 with $L_{0}$ replacing $L$ and $\Omega$ replacing $G$.

In what follows $N$ will be chosen so large that $B_{N}$ contains $\partial G$ in its interior. The corresponding $\omega^{N}$ will be denoted by $\omega$. Moreover, we define $M_{L_{0}}$, $\left(M_{L_{0}}^{*}\right)$ analogously as $M_{L}\left(M_{L}^{*}\right)$ with $\Omega=G \cap B_{3 N}$ replacing $G$ and $L_{0}$ replacing $L$. We need the following

3.7. Lemma. Let $G$ be a bounded domain with a $C^{1,1}$ boundary. Then $M_{L}=T_{L}$ and $M_{L}^{*}=T_{L *}$.

Proof. By Green's formulas it is clear that $M_{L} \supseteqq T_{L}$ and $M_{L}^{*} \supseteqq T_{L *}$. But since all the operators in question are closed and $T_{L *}=\left(T_{L}\right)^{*} \supseteqq$ $M_{L}^{*} \supseteq T_{L}^{*}$ and as a consequence $T_{L *}=M_{L *}$, it also follows that $T_{L}=$ $\left(T_{L *}\right)^{*}=M_{L}^{* *}=M_{L}$.

We return now to unbounded regions.

3.8. Lemma. Let $v \in \mathscr{D}\left(M_{L}\right)\left(\mathscr{D}\left(M_{L}^{*}\right)\right)$, then $\omega v$ restricted to $\Omega$ is in $\mathscr{D}\left(M_{L_{0}}\right)\left(\mathscr{D}\left(M_{L_{0}}^{*}\right)\right)$.

Proof. By Theorem 3.5, $\omega v \in W^{1,2}(G)$ and $-\Delta(\omega v)$ is a distribution in $L^{2}(G)$. The same is also true therefore if $G$ is replaced by $\Omega$. It suffices to show therefore that for $u \in C_{0}^{\infty}\left(E^{n}\right)$

$$
(u,-\Delta(\omega v))_{\Omega}=B_{\Omega}(u, \omega v) \text {. }
$$

The proof of this is similar to that of the corresponding statement of Theorem 3.5 and will be omitted.

3.9. Theorem. $M_{L}=T_{L}$ and $M_{L}^{*}=T_{L *}$. Thus $\left(T_{L}\right)^{*}=T_{L *}$.

Proof. By Lemma 3.8, $\omega v \in \mathscr{D}\left(M_{L_{0}}\right)$ whenever $v \in \mathscr{D}\left(M_{L}\right)$. Thus by Lemma 3.7, $\omega v \in \mathscr{D}\left(T_{L}\right)$. It then follows that $v \in \mathscr{D}_{1}(G)$ and $\widetilde{v}_{n}-$ $L \widetilde{v}=0$, since $\omega=1$ on $B_{N} \cap G$. Thus $v \in \mathscr{D}\left(T_{L}\right)$ and $M_{L} v=T_{L} v$ and the result follows. The proof that $M_{L}^{*}=T_{L *}$ is similar and will be omitted. It is clear from Theorem 2.7 that the spectrum of $T_{L}$ lies in a half plane. We can sharpen this estimate considerably to obtain the results of [1].

3.10. THEOREM. The spectrum of $T_{L}$ lies inside the the parabolic region $\tau^{2}=a \sigma+b$ with $a, b>0$ where a complex number $\lambda=\sigma+i \tau$.

Proof. We follow very closely the proof of Theorem 4.14 of [1] 
first recalling the definition of the operators $S, \hat{L}$ and $P(\lambda) . \quad \mathscr{D}(S)=$ $\left\{[u, \widetilde{u}]: u \in \mathscr{D}_{1}(G)\right\}$ and $S[u, \widetilde{u}]=\left[-\Delta u, \widetilde{u}_{n}\right]$ for $[u, \widetilde{u}] \in \mathscr{D}(S) . \quad \hat{L}$ and $P(\lambda)$ have all of $L^{2}(G) \oplus L^{2}(\partial G)$ as domains and $\hat{L}[f, g]=[0, L g), P(\lambda)[f, g]=$ $[\lambda f, 0]$. It was shown in [1] that when $\lambda$ is such that

$$
|([S-P(\lambda)][u, \widetilde{u}],[u, \widetilde{u}])|>\|L\|\|[u, \widetilde{u}]\|^{2},
$$

then zero is in the resolvent set of $S-\hat{L}-P(\lambda)$, and, moreover, for $[u, \tilde{u}] \in \mathscr{D}(S)$

$$
|([S-\hat{L}-P(\lambda)][u, \widetilde{u}],[u, \widetilde{u}])| \geqq\left. K(\lambda)||[u, \widetilde{u}]\right|^{2},
$$

where $K(\lambda)$ is a positive number.

But for $[u, \tilde{u}] \in \mathscr{D}(S)$

$$
([S-\hat{L}-P(\lambda)][u, \widetilde{u}],\lfloor u, \widetilde{u}])=\|\nabla u\|^{2}-\lambda\|u\|^{2}-(L \widetilde{u}, \widetilde{u})_{\partial}
$$

and for $u \in \mathscr{D}\left(T_{L}\right)$

$$
\left(\left(T_{L}-\lambda\right) u, u\right)=\|\nabla u\|^{2}-\lambda\|u\|^{2}-(L \tilde{u}, \widetilde{u})_{\partial} .
$$

Thus if (3.10.2) holds for some $K(\lambda)$, then for the same $K(\lambda)$

$$
\left|\left(\left(T_{L}-\lambda I\right) u, u\right)\right| \geqq K(\lambda)\|u\|^{2}, \quad u \in \mathscr{D}\left(T_{L}\right) .
$$

It only remains to determine conditions on $\lambda$ such that (3.10.1) is valid. At this point we may proceed exactly as was done in [1] and we arrive at the same conclusions.

4. Regions with unbounded boundaries. As was remarked in the introduction we shall restrict $L$ to be multiplication by a bounded measurable function. We shall then use a result of Poulsen [11] to obtain most of the results of the preceding section.

4.1. Definition. The operator $M_{L}$, when $L$ is the zero operator, will be denoted by $N . N$ is the operator corresponding to the Neumann boundary condition.

4.2 Theorem. Suppose $\omega \in C_{0}^{1,1}\left(E^{n}\right)$ is real valued and such that $|\omega|,|\nabla \omega|$, and $|\Delta \omega|$ are all uniformly bounded on $G$ by say $K$. Then if $\omega \in \mathscr{D}(N)$ and $v \in \mathscr{D}\left(M_{L}\right)\left(\mathscr{D}\left(M_{L *}\right)\right)$, if follows that $\omega v$ also in $\mathscr{D}\left(M_{L}\right)\left(\mathscr{D}\left(M_{L *}\right)\right)$.

Proof. Let $B_{N}$ be a sphere with center at the origin and radius $N$ so large that it contains the support of $\omega$ in the interior. $\Omega=G \cap B_{N}$ and observe that as in the proof of Theorem 3.5, it suffices to show that for all $u \in C_{0}^{2}\left(E^{n}\right)$ 


$$
(u, \Delta(\omega v))=B(u, \omega v)
$$

The proof is almost identical to that of Theorem 3.5. We observe first that if the support of $\omega$ is contained in $G$ we may simply apply Green's formulas. If the support of $\omega \cap \partial G$ is not empty we follow the arguments of Theorem 3.5. Note that since $\omega \in \mathscr{D}(N)$ and $u \in C_{0}^{2}\left(E^{n}\right)$ we find that $(u, v(-\Delta \omega))=\int_{a} \nabla(u \bar{v}) \cdot \nabla \omega d x$.

Following the computations in the proof of Theorem 3.5 yields (4.2.1). The proof for $M_{L}^{*}$ is almost identical.

4.3. Lemma. (Poulsen [11]). Let $G$ be a uniformly $C^{3}$ domain and let $\theta$ be any point in $\partial G$. There exist neighborhoods $A_{1}$ and $A_{2}$ of $\theta$ with compact closures such that $\bar{A}_{1} \subseteq A_{2}$ Moreover, there exists a real valued function $\omega \in C_{0}^{2}\left(E^{n}\right)$ such that $\omega$ is identically 1 in $G \cap A_{1}$ and vanishes in $G \cap A_{2}^{c}$. Moreover, $\omega \in \mathscr{D}(N)$ and $|\omega|,|\nabla \omega|$, and $|\Delta \omega|$ are uniformly bounded on $G$.

4.4. Definition. Let $\mathscr{D}\left(T_{L}\right)$ be the set of those functions in $\mathscr{D}_{1}(G)$ for which $\widetilde{u}_{n}-L \widetilde{u}=0$ on every compact subset of $\partial G$. For $u \in \mathscr{D}\left(T_{L}\right)$ let $T_{L} u=-\Delta u$.

\subsection{Lemma. $T_{L} \subseteq M_{L}$ and $T_{L *} \subseteq M_{L *}$.}

Proof. As usual we note that it suffices to show that for every $u \in C_{0}^{2}\left(E^{n}\right),(u,-\Delta v)=B_{G}(u, v)$.

We give the proof for $T_{L}$, the proof for $T_{L *}$ being similar. We note that $G$ need only be a $C^{1,1}$ manifold for this proof. As usual let $B_{N}$ be a sphere with center at the origin and with radius $N$ sufficiently large that for a fixed $u \in C_{0}^{2}\left(E^{n}\right)$, support of $u$ is contained in $B_{N}$. Let $\Omega=B_{N} \cap G$. Then

$$
\begin{aligned}
\int_{a} u(-\overline{\Delta v}) d x & =\int_{\Omega} u(-\overline{\Delta v}) d x=\int_{\Omega} \nabla u \cdot \overline{\nabla v} d x-\int_{\partial \Omega} \tilde{u} \overline{\widetilde{v}}_{n} d \theta \\
& =\int_{\Omega} \nabla u \cdot \overline{\nabla v} d x-\int_{\partial \Omega \cap \partial \theta} \tilde{u} \overline{\widetilde{v}}_{n} d \theta \\
& =\int_{a} \nabla u \cdot \overline{\nabla v} d x-\int_{\partial G} \tilde{u} \overline{\widetilde{v}}_{n} d \theta .
\end{aligned}
$$

Since $u$ was arbitrary in $C_{0}^{1,1}\left(E^{n}\right)$ the result follows.

Referring to Definition 1.2, let $V_{k}$ be one of the covering neighborhoods so that $T_{k}\left(V_{k} \cap G\right)=\Gamma \times\left\{0<x_{n}<r\right\}$. Now let $S_{1}, S_{2}$, and $S_{3}$ be three $C^{3}$ manifolds such that

$$
S_{1} \supseteq \frac{1}{2} \Gamma \times \overline{\left\{0<x_{n}<r / 2\right\}}
$$




$$
\begin{aligned}
& S_{2} \supseteqq \bar{S}_{1} \\
& S_{3} \supseteqq \bar{S}_{2} \\
& \Gamma \times\left\{0<x_{n}<r\right\} \supseteqq \bar{S}_{3} .
\end{aligned}
$$

Let $A_{i k}=T_{k}^{-1}\left(S_{i}\right)$ for $i=1,2,3$ and observe that $\bigcup_{k} A_{i k}$ contains an $r / 2$ neighborhood of $\partial G$. Now let $\omega$ be the function of Lemma 4.3 with $A_{1 k}$ and $A_{2 k}$ playing the roles of $A_{1}$ and $A_{2}$.

Let $\partial A_{3 k} \cap \partial G=\Gamma_{1}$ and $\Gamma_{2}=\partial A_{3 k} \sim \Gamma_{1}$.

We define an operator on $\partial A_{3 k}$ by

$$
L_{0} f(\theta)=\left\{\begin{array}{ll}
L f(\theta) & \text { for } \theta \in \Gamma_{1} \\
0 & \text { for } \theta \in \Gamma_{2}
\end{array} .\right.
$$

Let $M_{L_{0}} T_{L_{0}}$ be the operator corresponding to $L_{0}$ and $A_{3 k}$ as $M_{L}$ and $T_{L}$ correspond to $L$ and $G$. Then

4.6. Lemma. Let $v \in \mathscr{D}\left(M_{L}\right)\left(\mathscr{D}\left(M_{L *}\right)\right)$, then $\omega v \in \mathscr{D}\left(M_{L_{0}}\right)\left(\mathscr{D}\left(M_{L_{j}^{*}}\right)\right)$

Proof. The proof is almost identical to that of Theorem 4.2. By $\omega v$ we mean of course $\omega v$ restricted to $A_{3 k}$.

Now $A_{3 k}$ is a bounded $C^{3}$ manifold and thus by Lemma 3.8, $M_{L_{0}}=$ $T_{L_{0}}$ and $M_{L_{0}^{*}}=T_{L_{0}^{*}}$. Thus $\omega v$ is in $\mathscr{D}\left(T_{L_{0}}\right)$. Hence on $\Gamma_{1},(\omega v)_{n}-$ $L(\omega v)=0$. Thus in particular on $\partial G \cap \partial A_{i k}, \widetilde{v}_{n}-L \widetilde{v}=0$, since here $\omega$ is identically one. Since $\cup A_{i k}$ covers an $r / 2$ neighborhood of $\partial G$, it follows that $\widetilde{v}_{n}-L \widetilde{v}=0$ on every compact subset of $\partial G$. Combining these facts with Lemma 4.5, we finally obtain

4.7. THEorem. If $G$ is a uniformly $C^{3}$ manifold then $T_{L}=M_{L}$ and $T_{L *}=M_{L *}$. Thus $\left(T_{L}\right)^{*}=T_{L *}$.

The fact that $T_{L *}=M_{L *}$ follows by using almost the same argument as the above.

\section{REFERENCES}

1. W. G. Bade and R. S. Freeman, Closed extensions of the Laplace operator determined by a general class of boundary conditions. (Submitted for publication.)

2. F. E. Browder, On the regularity properties of elliptic differential equations, Comm. Pure Appl. Math., 9 (1956), 351-361.

3. - (Forthcoming publication.)

4. J. W. Calkin, Abstract symmetric boundary conditions, Trans. Amer. Math. Soc., 45 (1939), 369-442.

5. G. Ehrling, On a type of eigenvalue problem for certain elliptic differential operators, Math. Scand., 2 (1954), 267-285.

6. K. O. Friedrichs, Spektraltheorie halbbeschränkter Operatoren und Anwendung auf 
die Spektralzerlegung von Differentialoperatoren I, II, Math. Ann., 109 (1934), 465-486, 685-713.

7. Lars Hörmander, On the theory of general partial differential operators, Acta Math., 94 (1955), 161-248.

8. P. D. Lax and A. N. Milgram, Parabolic equations, contributions to the theory of partial differential equations, Ann. Math. Studies, No. 33, Princeton University Press (1954), 167-190.

9. K. R. Lucas. Submanifolds of dimension $n-1$ in $E^{n}$ with normals satisfying a Lipschitz condition, Studies in eigenvalue problems, Technical Report 18, University of Kansas, 1957.

10. L. Nirenberg, Estimates and existence of solutions of elliptic equations, Comm. Pure Appl. Math., 9 (1956), 509-530.

11. E. T. Poulsen, Some results on degenerate linear elliptic differential operators of the second order, Technical Report No. 25, Contract Nonr-222 (37) University of California, Berkeley, 1958.

LAWRENCE RAdiation Laboratory, University of CALIFornia

LIVERMORE, CALIFORNIA 



\section{PACIFIC JOURNAL OF MATHEMATICS}

\section{EDITORS}

Ralph S. Phillips

Stanford University

Stanford, California

M. G. Arsove

University of Washington

Seattle 5, Washington
A. L. Whiteman

University of Southern Californla

Los Angeles 7, California

Lowell J. Paige

University of California

Los Angeles 24, California

\section{ASSOCIATE EDITORS}

E. F. BECKENBACH

D. DERRY

H. L. ROYDEN

E. G. STRAUS

T. M. CHERRY

M. OHTSUKA

E. SPANIER

F. WOLF

\section{SUPPORTING INSTITUTIONS}

UNIVERSITY OF BRITISH COLUMBIA

STANFORD UNIVERSITY

CALIFORNIA INSTITUTE OF TECHNOLOGY

UNIVERSITY OF CALIFORNIA

MONTANA STATE UNIVERSITY

UNIVERSITY OF TOKYO

UNIVERSITY OF UTAH

UNIVERSITY OF NEVADA

NEW MEXICO STATE UNIVERSITY

OREGON STATE UNIVERSITY

UNIVERSITY OF OREGON

OSAKA UNIVERSITY

WASHINGTON STATE UNIVERSITY

UNIVERSITY OF WASHINGTON

UNIVERSITY OF SOUTHERN CALIFORNIA

AMERICAN MATHEMATICAL SOCIETY CALIFORNIA RESEARCH CORPORATION SPACE TECHNOLOGY LABORATORIES NAVAL ORDNANCE TEST STATION 


\section{Pacific Journal of Mathematics}

\section{Vol. 12, No. $1 \quad$ January, 1962}

Jonathan L. Alperin, Groups with finitely many automorphisms $\ldots \ldots \ldots \ldots \ldots \ldots \ldots \ldots$

Martin Arthur Arkowitz, The generalized Whitehead product ................ 7

John D. Baum, Instability and asymptoticity in toplogical dynamics . . . . . . . . . . 25

William Aaron Beyer, Hausdorff dimension of level sets of some Rademacher series .... $\quad 35$

Frank Herbert Brownell, III, A note on Cook's wave-matrix theorem . . . . . . . . . . . . . 47

Gulbank D. Chakerian, An inequality for closed space curves ................. 53

Inge Futtrup Christensen, Some further extensions of a theorem of Marcinkiewicz ....... 59

Charles Vernon Coffman, Linear differential equations on cones in Banach spaces . . . . . 69

Eckford Cohen, Arithmetical notes. III. Certain equally distributed sets of integers . . . . . 77

John Irving Derr and Angus E. Taylor, Operators of meromorphic type with multiple poles

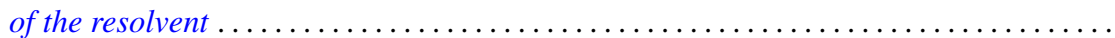

Jacob Feldman, On measurability of stochastic processes in products space .............

Robert S. Freeman, Closed extensions of the Laplace operator determined by a general class of boundary conditions, for unbounded regions ......................

Robert E. Fullerton, Geometric structure of absolute basis systems in a linear topological

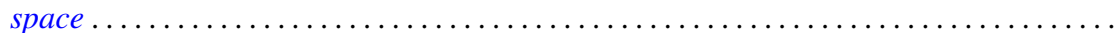

Dieter Gaier, On conformal mapping of nearly circular regions

Andrew Mattei Gleason and Hassler Whitney, The extension of linear functionals defined

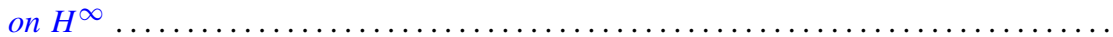

Seymour Goldberg, Closed linear operators and associated continuous linear

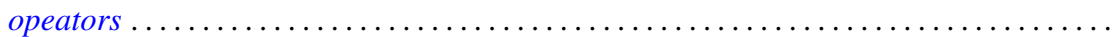

Basil Gordon, Aviezri Siegmund Fraenkel and Ernst Gabor Straus, On the determination of sets by the sets of sums of a certain order

Branko Grünbaum, The dimension of intersections of convex sets. .

Paul Daniel Hill, On the number of pure subgroups

Robert Peter Holten, Generalized Goursat problem . .

Alfred Horn, Eigenvalues of sums of Hermitian matrices ...........

Henry C. Howard, Oscillation and nonoscillation criteria for

$$
y^{\prime \prime}(x)+f(y(x)) p(x)=0
$$

Taqdir Husain, $S$-spaces and the open mapping theorem ...

Richard Eugene Isaac, Markov processes and unique stationary probability measures ...

John Rolfe Isbell, Supercomplete spaces ....................

John Rolfe Isbell, On finite-dimensional uniform spaces. II .........

N. Jacobson, A note on automorphisms of Lie algebras ..............

Antoni A. Kosinski, A theorem on families of acyclic sets and its applications

Marvin David Marcus and H. Minc, The invariance of symmetric functions of singular values...

Ralph David McWilliams, A note on weak sequential convergence.

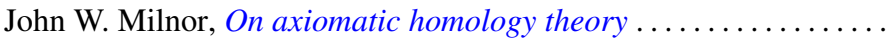

Victor Julius Mizel and Malempati Madhusudana Rao, Nonsymmetric projections in

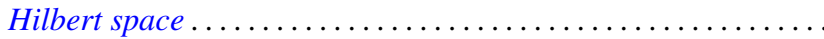

Calvin Cooper Moore, On the Frobenius reciprocity theorem for locally compact

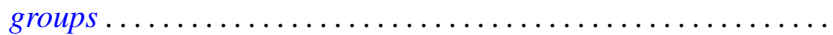

Donald J. Newman, The Gibbs phenomenon for Hausdorff means . 